\title{
Correlation of rheoencephalography and laser Doppler flow: a rat study
}

\author{
Michael Bodo ${ }^{1,4}$, Ryan Sheppard ${ }^{2}$, Aaron Hall ${ }^{2}$, Martin Baruch ${ }^{3}$, Melissa Laird ${ }^{2}$, Shravalya Tirumala ${ }^{2}$, \\ Richard Mahon ${ }^{2}$ \\ 1. Departments of Neurology and Surgery, Uniformed Services University of the Health Sciences, Bethesda, MD, USA \\ 2. Naval Medical Research Center, Silver Spring, MD; USA \\ 3. Empirical Technologies Corporation, Charlottesville, VA, USA \\ 4. E-mail any correspondence to: mikebodo@comcast.net
}

\begin{abstract}
Measuring brain electrical impedance (rheoencephalography) is a potential technique for noninvasive, continuous neuro-monitoring of cerebral blood flow autoregulation in humans. In the present rat study, we compared changes in cerebral blood flow autoregulation during $\mathrm{CO}_{2}$ inhalation measured by rheoencephalography to changes measured by laser Doppler flowmetry, an invasive continuous monitoring modality. Our hypothesis was that both modalities would reflect cerebral blood flow autoregulation.

Male Sprague-Dawley rats $\left(\mathrm{n}=28 ; 28\right.$ control and $82 \mathrm{CO}_{2}$ challenges) were measured under anesthesia. The surgical preparation involved implantation of intracerebral REG electrodes and an LDF probe into the brain. Analog waveforms were stored in a computer.

$\mathrm{CO}_{2}$ inhalation caused transient, simultaneous increases in the signals of both laser Doppler flow $(171.99 \pm 46.68 \%)$ and rheoencephalography $(329.88 \pm 175.50 \%)$. These results showed a correlation between the two measured modalities; the area under the receiver operating characteristic curve was 0.8394 .

The similar results obtained by measurements made with laser Doppler flowmetry and rheoencephalography indicate that rheoencephalography, like laser Doppler flowmetry, reflects cerebral blood flow autoregulation. Rheoencephalography therefore shows potential for use as a continuous neuro-monitoring technique.
\end{abstract}

Keywords: neuro-monitoring, laser Doppler flow, rheoencephalogram, $\mathrm{CO}_{2}$ inhalation, animal study (rat)

\section{Introduction}

Applications of rheoencephalography for monitoring cerebral blood flow autoregulation

The goal of neuromonitoring in both the neurosurgery intensive care unit, during transport of wounded military service members is to prevent brain damage that may occur due to failure of CBF AR. Currently, there is no single measuring modality capable of monitoring for such brain injuries as hypoxia, ischemia, elevated ICP, edema, intracranial hemorrhage, vasospasm.

CBF AR reflects the ability of the brain to keep brain blood flow relatively constant despite changes in arterial pressure. Failure of autoregulation is associated with a worse outcome in various acute neurological diseases [5]. The suitability of REG as a potential brain monitoring modality is due to its potential to detect vasoreactivity. Continuous $\mathrm{CBF}$ AR assessment would have prognostic value, indicate pathological states, and potentially influence therapy with the use of the 'optimal cerebral perfusion pressure' paradigm (CPP) [6]. Pressure autoregulation is an important hemodynamic mechanism, which protects the brain against inappropriate fluctuations in CBF in the face of changing CPP. Pressure autoregulation can be monitored with ICM+ software [2].

Few studies have compared results of measurements made with REG to LDF measurements.

Our study established a correlation between REG and LDF during transient $\mathrm{CO}_{2}$ inhalation. The physical basis of REG is that blood and cerebrospinal fluid are better conductors than brain tissue. Consequently, increases in REG amplitude reflect increases in the amount of blood in the brain. The usefulness of REG for neuromonitoring compared to LDF is that (when used to measure humans), REG is noninvasive. A disadvantage of REG is its sensitivity to artifacts; to overcome this disadvantage in our study, we used data pre-processing to clean the REG signal before quantification (see Methods).

This study is part of an ongoing investigation of brain electrical impedance (rheoencephalography/REG) and its potential for use as a noninvasive, continuous brain monitor. The suitability of REG for neuro-monitoring is due to its capability to detect vasoreactivity, the change in cerebral blood flow autoregulation (CBF AR) in response to a vasodilatory or vasoconstrictive stimulus. $\mathrm{CBF} A \mathrm{AR}$ is an instrinsic neuroprotective physiological phenomenon, which reflects the ability of the brain to maintain relatively constant $\mathrm{CBF}$ despite changes in cerebral perfusion pressure. This study compared results measured simultaneously by REG and laser Doppler flowmetry (LDF), a commonly used neuromonitoring modality. Using LDF, Lee et al. showed that breaching lower limits of autoregulation was associated with a significant increase of the pressure reactivity index $(\mathrm{PRx})$ in a model of arterial hypotension [9]. The rat study reported here measured $\mathrm{CBF}$ AR during $\mathrm{CO}_{2}$ inhalation (a vasodilatory stimulus).

A strong correlation between cerebrovascular reactivity (CVR) and ventilatory response to $\mathrm{CO}_{2}$ has been demonstrated [1]. $\mathrm{CBF}$ and its distribution are highly sensitive to changes in the partial pressure of arterial $\mathrm{CO}_{2}$ 
$\left(\mathrm{pCO}_{2}\right)$. This physiological response (cerebrovascular $\mathrm{CO}_{2}$ reactivity) is a vital homeostatic function that helps regulate and maintain central $\mathrm{pH}$, which in turn affects the respiratory central chemoreceptor stimulus. As a response to hypercapnia, $\mathrm{CBF}$ increases to wash out $\mathrm{CO}_{2}$ from brain tissue, thereby attenuating the rise in central $\mathrm{pCO}_{2}$; in contrast, hypocapnia causes cerebral vasoconstriction, which reduces $\mathrm{CBF}$ and attenuates the fall of brain tissue $\mathrm{pCO}_{2}$ [1]. Carbon dioxide is the most suitable vasoactive stimulus [7]. Acetazolamide and $\mathrm{CO}_{2}$-induced stimulation of the cerebral vasomotors have been shown to be valid techniques to measure reduction in perfusion reserve due to extracranial cerebrovascular occlusive disease [12].

Our hypothesis was that both LDF and REG reflect CBF AR.

\section{Methods}

The methods and protocols reported here were reviewed and approved by the Institutional Animal Care and Use Committee.

Each of twenty eight male Wistar rats was anesthetized (ketamine/xylazine ip anesthesia) and exposed to three $\mathrm{CO}_{2}$ inhalation challenges $(n=82)$.

Brain blood flow was recorded with a laser Doppler flowmeter (PeriFlux 4001, Perimed, Jarfalla, Sweden). REG amplifiers were Cerberus (Quintlab, Budapest, Hungary) and an EEG/REG amplifier (Empirical Technologies Corporation, Charlottesville, VA). Each rat inhaled $15-20 \% \mathrm{CO}_{2}$ gas for 30 seconds. Gas was mixed with $21 \%$ $\mathrm{O}_{2}$, balanced nitrogen using a gas mixer (Pegas $4000 \mathrm{MF}$, Columbus Instruments, Columbus, $\mathrm{OH}$ ) from gas tanks (GT \& $\mathrm{S}$, Allentown, PA). Exhaled $\mathrm{CO}_{2}$ concen-tration was measured with $\mathrm{CO}_{2}$ analyzer (Capstar-100, CWE Inc, Ardmore, PA). The calibration gas was $5 \% \quad \% \quad \mathrm{CO}_{2}$ (Compressed gas, $5 \% \mathrm{CO} 2,21 \% \mathrm{O}_{2}$, balance nitrogen; Scott Medical Product, Plumsteadville, PA).

Signals were stored in a Dell computer equipped with an analog/digital converter card with 16 bit resolution (PCIMIO-16XE-10, National Instruments, Austin, TX); measurement software was developed by the Walter Reed Army Institute of Research (DataLyser). Data were acquired at a $200 \mathrm{~Hz}$ sampling rate.

The head of each rat was secured by a gas anesthesia head holder (designed for rats) of the stereotaxic frame (David Kopf Instruments, Tujunga, CA). The skin was removed to expose the cranium between the sutura frontalis and the sutura lambda. The exposed bone was rinsed with hydrogen peroxide in preparation for the application of acrylic adhesive. Four burr holes $(0.8 \mathrm{~mm})$ were drilled for insertion of intracranial electrodes (E 363/1, Plastics One, Roanoke, VA), two holes for EEG (left side) and two for REG (right side). Intracerebral electrodes were positioned 2 $\mathrm{mm}$ in front of the sutura lambdoidea and $2 \mathrm{~mm}$ lateral to the sutura sagittalis (Fig. 1). Frontal electrodes were placed $2 \mathrm{~mm}$ in front of the sutura coronalis. Inter-electrode distance was $6 \mathrm{~mm}$ and $10 \mathrm{~mm}$. REG electrodes were placed at a $5 \mathrm{~mm}$ depth, perpendicular to the surface of the skull; REG electrode diameter was $0.35 \mathrm{~mm}$; length of uninsulated electrode tips was $5 \mathrm{~mm}$. For the LDF probe, a burr hole was drilled (2.3 $\mathrm{mm}$ bit) between the REG electrodes. The EEG ground was inserted in neck muscle tissue. Next, electrodes were fixed to the skull, first with instant adhesive (454 Loctite, Hartford, CT) then with dental acrylate (Plastics One, Roanoke, VA). Electrodes were then connected to cables and to EEG and REG amplifiers. Additional amplifier channels were used for recording electroencephalograms, electrocardiograms, and respiration by thoracic impedance.

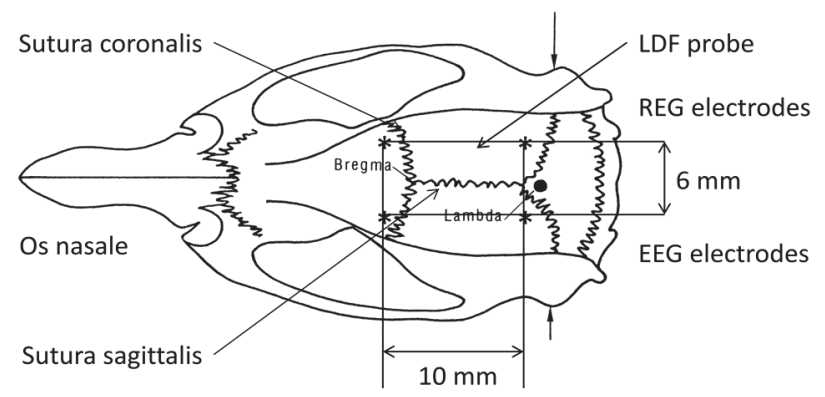

Fig. 1. Anatomical positions of REG, EEG electrodes and LDF probe are shown implanted in a rat skull (dorsal view). Standard orientation points in rat neurophysiology: bregma and lambda points and sutura between bones.

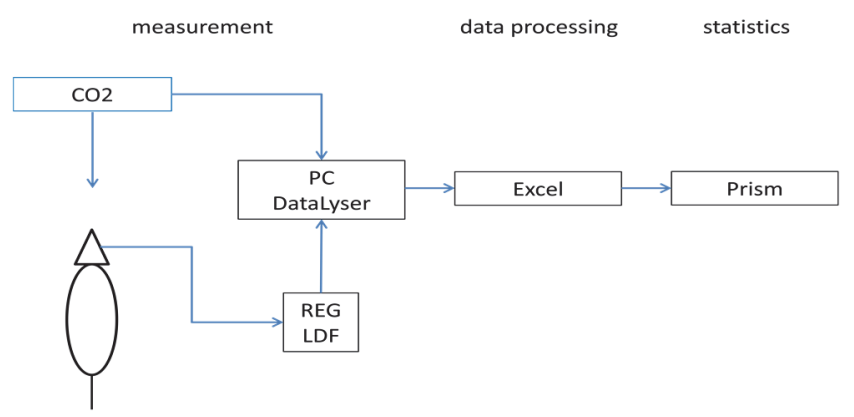

Fig. 2. Block schematics showing REG and LDF measured during rat $\mathrm{CO}_{2}$ inhalation challenges and data processing of resulting signals stored in a computer (PC). Data were first processed using DataLyser software to process analog signals; numbers generated were then put into an Excel software spread sheet for calculation of percentages of control values, group mean and SD. Next, percent values of REG and LDF were put into a Prism software spreadsheet for calculation of ROC and AUC.

Data processing was performed with DataLyser (Fig 2). Respiratory subharmonics were removed with a $2 \mathrm{~Hz}$ highpass Chebyshev filter. Noise was removed $(0.1 \mathrm{~Hz}$ running average). The first derivative of REG was calculated, after which the signal was rectified, and a running integral was calculated (5 second window). For baseline (before $\mathrm{CO}_{2}$ 
inhalation) and during maximal amplitudes, $30 \mathrm{sec}$ periods were marked by cursors, and basic statistics were calculated. From these data, mean values were copied into an Excel spreadsheet. Each mean amplitude was represented as a percentage of the baseline of that specific challenge. Statistical analysis was performed by applying percentage values rather than mean amplitudes. GraphPad Prism software (La Jolla, CA) was used for statistical analysis (receiver operator characteristics - ROC).

\section{Results}

$\mathrm{CO}_{2}$ inhalation caused a transient, simultaneous increase in LDF flux and REG pulse amplitude (Fig. 3).

LDF increase was $171.99 \pm 46.68 \%$; REG increase was $329.88 \pm 175.50 \%$. LDF flux increase correlated with REG pulse amplitude increase. The area under the receiver operating characteristic curve was 0.8394 ; SE 0.0693 ; $95 \%$ conf. interval was 0.7670 to 0.9118 ; $\mathrm{p}<0.0001$ (Fig. 4).

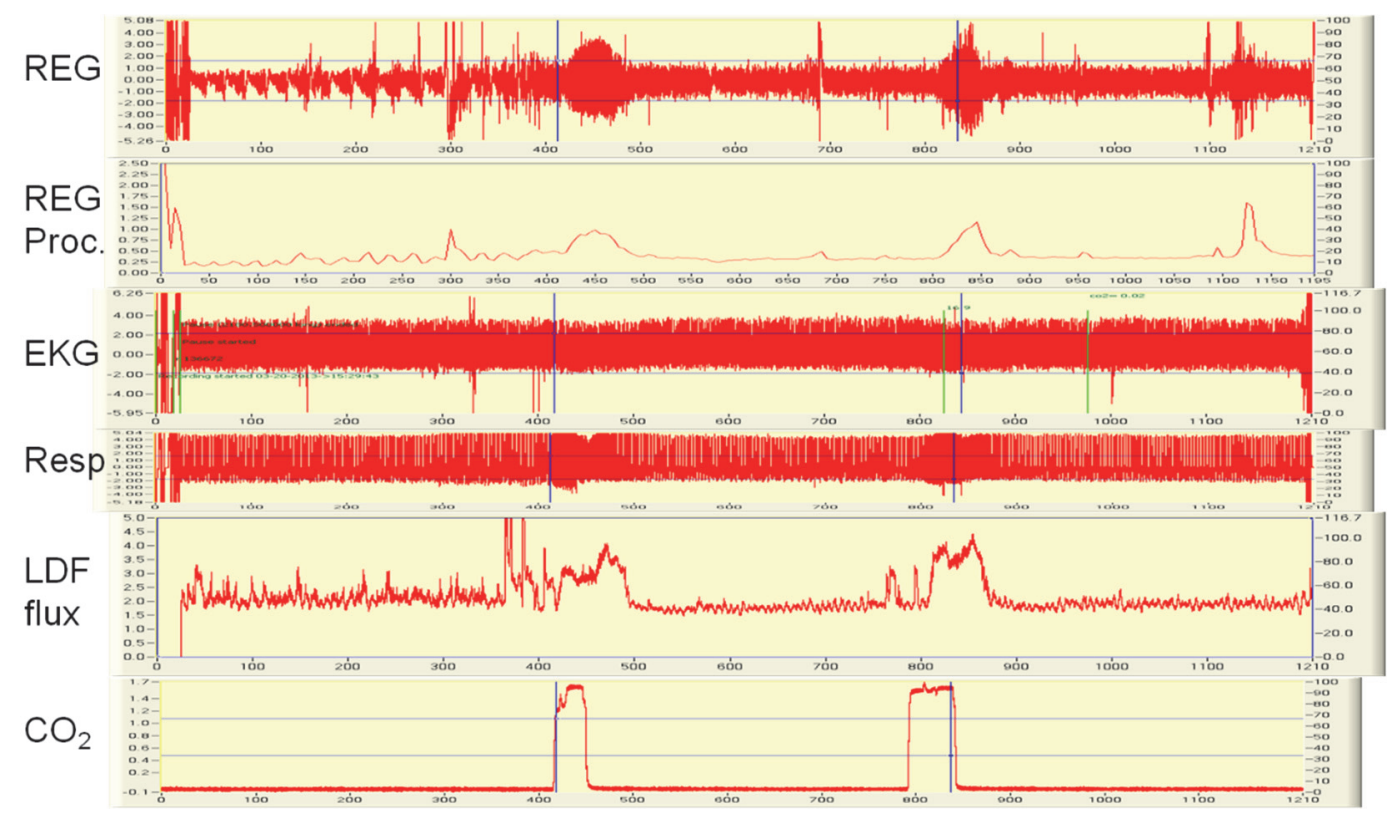

Fig. 3. Impact of $\mathrm{CO}_{2}$ inhalation on LDF flux and REG signals, in real time: $\mathrm{CO}_{2}$ increases were followed by simultaneous increases in LDF flux and REG signal amplitudes. For all traces, Y axis is shown in volts; X axis is shown in seconds. Traces, from top, are REG raw signal; REG proc (processed signal, displayed as a running integral); EKG; Respiration (measured by bioimpedance); $\mathrm{LDF}$ flux; inhaled $\mathrm{CO}_{2}$.

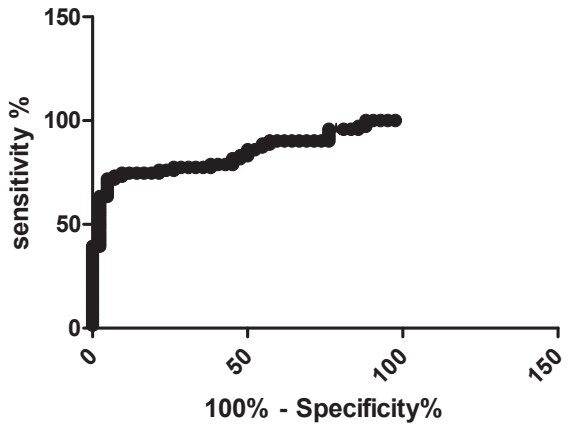

Fig. 4. ROC curve of LDF and REG. Area under curve (AUC): 0.8394 with $\mathrm{p}<0.0001$; Std. error: 0.03693 ; $95 \%$ confidence interval: 0.7670 to 0.9118 . Figure and numbers are presented as Prism program shown and calculated from the percent values of REG and LDF signals.

\section{Discussion}

This study is part of a series of ongoing measurements using rheoencephalography (REG) for neuromonitoring during physiological and pathological cerebral blood flow manipulations [3,4]. The purpose of our studies is to investigate the suitability of REG for use in developing a noninvasive, continuous brain monitor for medical, air and space applications.

\section{Brain monitoring modalities}

An international consensus has been published on the usefulness of multimodality monitoring in neurocritical care [10]. Invasive neuro-monitoring modalities typically measured are blood flow by LDF, intracranial pressure (ICP), brain tissue $\mathrm{O}_{2}$ and temperature, and tissue perfusion. Noninvasive modalities used are near infrared spectroscopy (NIRS), transcranial Doppler (TCD) and neurophysiological methods (EEG, evoked potentials).

Brain imaging methods such as single-photon emission computed tomography (SPECT), Positron Emission Tomography (PET) scan, Magnetic Resonance Imaging (MRI), and cerebral perfusion computed tomography are not suitable for continuous monitoring and detecting the onset of neurological deterioration. 
Why REG can be used for air and space research?

A NASA Technical Reports search for REG [11] found 90 publications containing REG as a keyword, most referring to bibliographies and indexes. However, a report related to REG from 1979 stated that there was an increase in the blood volume in the head that was retained for the entire 96-day flight and leveled off at the end the 140-day flight [17].

One of the reports describes that $+\mathrm{Gz}$ acceleration without straining maneuvers decreases $\mathrm{CBF}$ velocity to half of normal and probably caused a caudal fluid shift from both intra- and extracranial tissues [8]. A similar effect was described in a rabbit study [14]. Impaired cerebrovascular autoregulation and reduced $\mathrm{CO}_{2}$ reactivity after long duration spaceflight was reported [16].

\section{Conclusion}

The present study was conducted to acquire a fundamental understanding of the potential of REG for use in developing a non-invasive brain monitor capable of continuously assessing the integrity of CBF AR. The suitability of REG as a potential brain monitoring modality is due to its potential to detect vasoreactivity. REG has potential applications as a noninvasive, continuous neuromonitoring in the neurosurgery intensive care unit, during treatment and transport of wounded service members and in air and space research.

\section{Acknowledgements}

The authors thank Hanbing Zhou for technical assistance; Lajos Baranyi for developing and use of the DataLyser program; Diana Temple for administrative assistance; and Janice Meer for editorial services.

\section{Disclaimer}

The opinions expressed herein are those of the author(s), and are not necessarily representative of those of the Uniformed Services University of the Health Sciences (USUHS), the Department of Defense (DOD); or, the United States Army, Navy, or Air Force.

\section{References}

1. Ainslie PN, Duffin J. Integration of cerebrovascular $\mathrm{CO}_{2}$ reactivity and chemoreflex control of breathing: mechanisms of regulation, measurement, and interpretation. Am J Physiol Regul Integr Comp Physiol. 2009;296:R1473-95. https://doi.org/10.1152/ajpregu.91008.2008

2. Anomymous: $\mathrm{ICM}+\AA$ Software for Brain Monitoring in Neurological Intensive Care

3. Bodo M. Studies in rheoencephalography (REG). J Electr Bioimp. 2010;1:118-40. https://doi.org/10.5617/jeb.109
4. Brady K M, Mytar J O, Kibler K K, et al. Monitoring cerebrovascular pressure reactivity with rheoencephalography. J. Phys. Conf. Ser. 2010; 224012089 https://doi.org/10.1088/1742-6596/224/1/012089

5. Czosnyka M, Miller C. Monitoring of cerebral autoregulation. Neurocrit Care. 2014;21 Suppl 2:95-102. https://doi.org/10.1007/s12028-014-0046-0

6. Donnelly J, Aries MJ, Czosnyka M. Further understanding of cerebral autoregulation at the bedside: possible implications for future therapy. Expert Rev Neurother. 2015;15:169-85. https://doi.org/10.1586/14737175.2015.996552

7. Fierstra J, Sobczyk O, Battisti-Charbonney A, Mandell DM, Poublanc J, Crawley AP, Mikulis DJ, Duffin J, Fisher JA. Measuring cerebrovascular reactivity: what stimulus to use? J Physiol. 2013;591(Pt 23):5809-21. https://doi.org/10.1113/jphysiol.2013.259150

8. Kawai Y, Puma SC Hargens AR, Murthy G, Warkander D, Lundgren CE. Cerebral blood flow velocity and cranial fluid volume decrease during $+\mathrm{Gz}$ acceleration. Document ID: 20040089145. J Gravit Physiol. 1997;4:31-4.

9. Lee JK, Kibler KK, Benni PB, Easley RB, Czosnyka M, Smielewski P, Koehler RC, Shaffner DH, Brady KM. Cerebrovascular reactivity measured by near-infrared spectroscopy. Stroke. 2009;40:1820-6. https://doi.org/10.1161/STROKEAHA.108.536094

10. Le Roux P, Menon DK et al. The international multidisciplinary consensus conference on multimodality monitoring in neurocritical care: evidentiary tables: a statement for healthcare professionals from the neurocritical care society and the European society of intensive care medicine. Neurocrit Care. 2014;21 Suppl 2:297-361. https://doi.org/10.1007/s12028-014-0081-x

11. Nasa Web site research (April 8, 2015)

12. Nusbaum D, Clark J, Brady K, Kibler K, Sutton J, Easley RB. Alteration in the lower limit of autoregulation with elevations in cephalic venous pressure. Neurol Res. 2014;36:1063-71. https://doi.org/10.1179/1743132814Y.0000000397

13. Ringelstein EB, Van Eyck S, Mertens I. Evaluation of cerebral vasomotor reactivity by various vasodilating stimuli: comparison of $\mathrm{CO}_{2}$ to acetazolamide. J Cereb Blood Flow Metab. 1992;12:162-8. https://doi.org/10.1038/jcbfm.1992.20

14. Shender BS, Dubin SE. Contribution of cerebrospinal fluid to rheoencephalographic waveforms during hypoxic and $+\mathrm{Gz}$ stress. Aviat Space Environ Med. 1994;65:510-7.

15. Vajkoczy P, Roth H, Horn P, Lucke T, Thomé C, Hubner U, Martin GT, Zappletal C, Klar E, Schilling L, Schmiedek P. Continuous monitoring of regional cerebral blood flow: experimental and clinical validation of a novel thermal diffusion microprobe. J Neurosurg. 2000;93:265-74. https://doi.org/10.3171/jns.2000.93.2.0265

16. Zuj KA, Arbeille P, Shoemaker JK, Blaber AP, Greaves DK, $\mathrm{Xu}$ D, Hughson RL. Impaired cerebrovascular autoregulation and reduced $\mathrm{CO}_{2}$ reactivity after long duration spaceflight. Am J Physiol Heart Circ Physiol. 2012;302:H2592-8. https://doi.org/10.1152/ajpheart.00029.2012

17. Yegorov AD. Results of medical studies during long-term manned flights on the orbital Salyut 6 and Soyuz complex. NASA TM 1979;76014. 